\title{
Highly Efficient Base Editing in Viral Genome Based on Bacterial Artificial Chromosome Using a Cas9-Cytidine Deaminase Fused Protein
}

\author{
Ke Zheng ${ }^{1,2} \cdot$ Fang-Fang Jiang ${ }^{1} \cdot$ Le Su$^{1} \cdot$ Xin Wang $^{1} \cdot$ Yu-Xin Chen ${ }^{1} \cdot$ Huan-Chun Chen ${ }^{1} \cdot$ Zheng-Fei Liu $^{1}$ (D)
}

Received: 12 April 2019/Accepted: 9 September 2019/Published online: 2 December 2019

(c) Wuhan Institute of Virology, CAS 2019

\begin{abstract}
Viruses evolve rapidly and continuously threaten animal health and economy, posing a great demand for rapid and efficient genome editing technologies to study virulence mechanism and develop effective vaccine. We present a highly efficient viral genome manipulation method using CRISPR-guided cytidine deaminase. We cloned pseudorabies virus genome into bacterial artificial chromosome, and used CRISPR-guided cytidine deaminase to directly convert cytidine (C) to uridine (U) to induce premature stop mutagenesis in viral genes. The editing efficiencies were $100 \%$. Comprehensive bioinformatic analysis revealed that a large number of editable sites exist in pseudorabies virus (PRV) genomes. Notably, in our study viral genome exists as a plasmid in E. coli, suggesting that this method is virus species-independent. This application of base-editing provided an alternative approach to generate mutant virus and might accelerate study on virulence and vaccine development.
\end{abstract}

Keywords Pseudorabies virus (PRV) - Bacterial artificial chromosome (BAC) · Base-editing · CRISPR/Cas9 . Genome editing

\section{Introduction}

Programmable manipulation of gene facilitates the understanding of gene function and development of genetic engineered virus vaccine (Post et al. 1990; Gasiunas et al. 2012; Jinek et al. 2012; Bull 2015; Wang H et al. 2016). Viruses, as small infectious agents, can infect all types of life forms (Koonin et al. 2006). Due to rapid evolution and diverse viral transmission strategies, viruses are still serious threats to animal health and economy (Peiris et al. 2003; Meyer and Wilke 2015; Ge et al. 2018; Zhou et al. 2018). Traditionally, editing in viral genome is based on

Electronic supplementary material The online version of this article (https://doi.org/10.1007/s12250-019-00175-4) contains supplementary material, which is available to authorized users.

Zheng-Fei Liu

1zf6789@mail.hzau.edu.cn

1 State Key Laboratory of Agricultural Microbiology and Key Laboratory of Preventive Veterinary Medicine in Hubei Province, College of Veterinary Medicine, Huazhong Agricultural University, Wuhan 430070, China

2 Gene Editing Research Center, Hebei University of Science and Technology, Shijiazhuang 050018, China spontaneous homologous recombination in host mammalian cells, or through lambda Red-mediated recombination at bacterial artificial chromosome (BAC) level in Escherichia coli (E. coli) (Adler et al. 2000), both of which are laborious. Therefore, efficient genome editing technologies in viral genome are still desired.

Even though the clustered regularly interspaced short palindromic repeats (CRISPR)/CRISPR-associated protein 9 (Cas9) system is employed to improve the recombination (Wang H et al. 2016), the generation of a genome-modified virus is still challenging and inconvenient due to the obstacles caused by virus. The obstacles include: (1) CRISPR/Cas9 system performed far from perfect when Cas9 protein meets rapidly increasing number of replicated viral genome DNAs; (2) manipulation in some genes could lower the replication rate of recombinant virus, making the selection task difficult; (3) reporter genes (e.g. green fluorescence protein, GFP) are still necessary for highly efficient purification of recombinant virus, which need to be removed for further study.

On the other hand, relying on lambda Red system, highly efficient homologous recombination allows simple generation of mutant virus at BAC level (Adler et al. 2000; Liu et al. 2008). The homologous sequences needed for 
recombination could be short as $30 \mathrm{bp}$, simplifying the preparation of donor DNA (Court et al. 2002). However, marker gene (antibiotics resistance gene) is still required for highly efficient selection. Thus, additional lambda Redmediated recombination needs to be performed in order to remove the marker gene.

Recently, CRISPR-guided precise base-editing technologies were developed (Komor et al. 2016; Ma et al. 2016; Nishida et al. 2016; Gaudelli et al. 2017). The "base editors" (BEs) mediate conversion of C.G to T.A (Komor et al. 2016; Ma et al. 2016; Nishida et al. 2016) or A.T to G.C (Gaudelli et al. 2017) within target region without double strand breaks (DSBs). The technologies rely on deamination reaction catalysed by cytidine deaminase or adenosine deaminase rather than homology-dependent repair (HDR), therefore donor DNA is not required. Since developed, BEs were widely used in mammalian cells, animals and plants (Komor et al. 2016; Billon et al. 2017; Kim et al. 2017a; Zong et al. 2017). Recent studies reported that the third generation $\mathrm{BE}$ (BE3) was used to induce premature stop (iSTOP) codon with high efficiency in Staphylococcus aureus, E. coli, and Brucella melitensis (Gu et al. 2018; Zheng et al. 2018).

Pseudorabies virus (PRV, Suid alphaherpesvirus 1), which is a member of the Alphaherpesvirinae subfamily of Herpesviridae, causes devastating disease (Aujeszky's disease) in swine, resulting in great economic losses in swine agriculture (Pomeranz et al. 2005). Recently, PRV variants emerged and caused infectious disease, posing a desperate demand for efficient viral genome editing technologies to facilitate virology study and vaccine development (Wu et al. 2013). To investigate these PRV variants, several researchers have cloned PRV-BACs for use as a rapid genome editing platform (Smith and Enquist 2000; Peng et al. 2009; Wang J et al. 2016, Wang et al. 2018). We envisioned that CRISPR-guided BE would enable rapid and efficient viral genome editing at BAC level in E. coli.

In this study, we presented a highly efficient method to edit viral genes using CRISPR-guided BE3 system based on BAC. We cloned PRV-Ea genome into BAC, and manipulated viral genes in E. coli cells. The induced premature mutagenesis in US8 and UL34 showed absolutely high efficiency (100\%). The US8 mutant virus was generated through reverse genetic technology and represent attenuation in intercellular transmission, which is consistent with gene function. In addition, a comprehensive bioinformatic analysis was performed to gain insight into distribution of iSTOPs in PRV variants. Taken together, our study provided a rapid and efficient approach to perform base-editing in viral genome at BAC level, which will facilitate study on virology.

\section{Materials and Methods}

\section{Virus Strain and Cell Line}

PRV-Ea strain was isolated in 1998 and replicated in our lab (Chen et al. 1998; Liu et al. 2002; Wang et al. 2017). PK-15 and MDBK cell lines were purchased from China Center for Type Culture Collection (CCTCC) and cultured in Dulbecco's modified Eagle's medium (Gibco) supplemented with $10 \%$ fetal bovine serum (Gibco).

\section{Construction of Transfer Vector pMD18-T-US9- US2-pHA2}

The mini-F plasmid pHA2 (Adler et al. 2000) was used as F-plasmid vector, which was designed to be inserted into intergenic region between US9 and US2. For insertion, a transfer vector based on pHA2 containing homologous arms was constructed. Briefly, the upstream homologous arm (include the partial US8 gene and the entire US9 gene) and downstream homologous arm (partial US2 gene) were amplified. After digestion by Hind III and Pac I for amplified upstream homologous arm and digestion by $P a c$ I and Sac I for amplified downstream homologous arm, the two fragments were ligated to pMD18-T digested by Hind III and Sac I, generating pMD18-T-US9-US2. After confirmation with digestion and Sanger sequencing, the plasmid was linearized with $P a c$ I and cloned into Pac I site of pHA2, resulting in transfer vector pMD18-T-US9US2-pHA2. The primers used are listed in Supplementary Table S1.

\section{Construction of a PRV BAC Clone}

The PRV-Ea genome DNA was extracted as previously described (Wang et al. 2017). The transfer vector pMD18T-US9-US2-pHA2 was linearized with Hind III and purified from agarose gel. To construct an infectious PRV BAC clone, $1.5 \mu \mathrm{g}$ of PRV-Ea genome DNA and $500 \mathrm{ng}$ of purified linearized pMD18-T-US9-US2-pHA2 were cotransfected into PK-15 cells using lipofectamine 2000 (Invitrogen). Since pHA2 backbone possesses $e G F P$ gene, the green fluorescence-positive plaques (recombinant PRV) were purified. Briefly, the plaques were picked using $100 \mu \mathrm{L}$ tips from PK-15 cells overlaid with $1 \%$ (w/v) lowmelting point agarose and 2\% FBS in DMEM. After four rounds of purification, all the plaques showed green fluorescence. A PRV-BAC recombinant was selected to infect PK-15 cells at a multiplicity of infection (MOI) of 5 to isolate the circular replicative form DNA. After $3 \mathrm{~h}$, the circular replicative-form PRV-BAC DNA was isolated and eletroporated into E. coli DH10B cells (Invitrogen) with 
$0.1 \mathrm{~cm}$ cuvettes at $1.8 \mathrm{kV}, 200 \Omega$, and $25 \mu \mathrm{F}$. After recovery, the transformed cells were then plated on Luria-Bertani (LB) agar plates containing $25 \mu \mathrm{g} / \mathrm{mL}$ chloramphenicol and incubated at $37{ }^{\circ} \mathrm{C}$ till colonies grew. The colonies were cultured in $5 \mathrm{~mL}$ LB medium containing $25 \mu \mathrm{g} / \mathrm{mL}$ chloramphenicol at $37^{\circ} \mathrm{C}$ shaking at $180 \mathrm{rpm}$ for $12 \mathrm{~h}$. The plasmids were extracted and confirmed by restriction fragment length polymorphism (RFLP) assay (Liu et al. 2008). Five microgram of DNA was digested with $\mathrm{BamH} \mathrm{I}$ at $37{ }^{\circ} \mathrm{C}$ for $2 \mathrm{~h}$, and electrophoresed on $0.5 \%$ $(\mathrm{w} / \mathrm{v})$ agarose gel in $1 \times \mathrm{TAE}$ buffer at $5 \mathrm{~V} / \mathrm{cm}$ electric field. Image was captured after staining with ethidium bromide.

\section{Construction of BE3 Plasmids}

The pEcBE3 was used for base-editing in E. coli (Zheng et al. 2018). To clone sgRNA into pEcBE3, a couple of oligonucleotides were annealed and ligated into linearized pEcBE3 digested with $B s a$ I. The ligation products were transformed into Mach1-T1 competent cells. The plasmids were confirmed using Sanger sequencing. Oligonucleotides and primers were listed in Supplementary Table S1.

\section{Base-Editing in pPRV-BAC}

The DH10B carrying pPRV-BAC cells were prepared into chemically competent cells. Base-editing was performed as described previously (Zheng et al. 2018). The pEcBE3 series plasmids were transformed into DH10B carrying pPRV-BAC competent cells. After heat-shock, transformed E. coli cells were incubated in SOC medium $(0.6 \mathrm{mmol} / \mathrm{L}$ IPTG) at $37{ }^{\circ} \mathrm{C}$ with shaking at 180 r.p.m. for $1 \mathrm{~h}$, and then spread on LB agar plate $(50 \mu \mathrm{g} / \mathrm{mL}$ ampicillin and 25 $\mu \mathrm{g} / \mathrm{mL}$ chloromycetin). To avoid satellite colonies contamination, single colony was isolated using T-streak method. Single colony was cultured, harvest and taken for plasmid extraction. The target regions were PCR amplified and sequenced to confirm mutagenesis.

\section{Rescue and Cre-Mediated Recombination of PRV}

PK-15 cells in cell culture dish with diameter of $35 \mathrm{~mm}$ were transfected with $1 \mu \mathrm{g}$ of pPRV-BAC plasmid and incubated till cytopathy occurred, thus generating rescued PRV mutants. To remove F-plasmid vector, the PK-15 cells in cell culture dish with diameter of $150 \mathrm{~mm}$ were transfected with $1 \mu \mathrm{g}$ of PRV-BAC genomic DNA and $1 \mu \mathrm{g}$ of pCAGGS-NLS/cre plasmid (Wang and Osterrieder 2011; Zou et al. 2014), and overlaid with $1.0 \%$ (w/v) low melting point agarose till PFU occurred. The rescued PRV (rPRV) was purified through plaque purification until no original PRV BAC recombinants was detected. The original PRV
BAC recombinants were detected using PCR amplification using primers P11 and P12 (Supplementary Table S1).

\section{Morphogeny Assay}

Viral titers (PFU/mL) were determined by plaque assays. MDBK monolayers were infected with 100 plaque-forming units (PFU) per well in six-well plates. The cells were then overlaid with $1.6 \%$ carboxymethyl cellulose for $48 \mathrm{~h}$, fixed with $10 \%$ formaldehyde, and finally stained by crystal violet. Images of discrete plaques per virus were acquired for analysis by a microscope equipped with a digital camera. Twenty-five plaques of each virus were analyzed. The average diameter for each plaque was calculated from two perpendicular diameter measurements performed using Adobe Acrobat 7.0 professional software.

\section{Growth Kinetics Assay}

One-step growth kinetics was conducted to compare the growth kinetics of the original strain PRV-Ea and PRVTgE mutant. PK-15 cell monolayer was infected with each virus at a MOI of 5. The cells were harvested and stored at $-80{ }^{\circ} \mathrm{C}$ after $0,3,12$ and $30 \mathrm{~h}$ post infection (hpi). Viral titer was determined by PFU.

\section{Genetic Stability Assay}

To investigate genetic stability of edited PRV infectious clone, the PRV-TgE mutant was sub-cultured for ten generation. Briefly, PK-15 cells were infected with PRV-TgE at MOI of 5 . After $24 \mathrm{~h}$, the cells were harvested and lysed through freezing and thawing for twice. One microliter of cell lysate was taken as template for PCR amplification and Sanger sequencing.

\section{Statistical Analysis}

Statistical analyses were performed using Prism version 7.03 for Windows (GraphPad Software, La Jolla, CA). Values are represented as means \pm standard deviations from independent experiments. Three independent experiments were performed. Statistical significance was evaluated using a two-tailed Student's $t$ test.

\section{Bioinformatics Analysis}

The genome sequences of PRV-Ea, PRV-Berker, and PRVHNX strains were obtained from NCBI (GenBank accession no. KX423960.1, JF797219, and KM189912, respectively). For CAA (Gln), CAG (Gln), and CGA (Arg) codons, the distance between editable codon and PAM should be 10-14 bp; for TGG (Trp) codon, the distance 
should be 10-15 bp. The distribution of iSTOP codons in genome was visualized by circos (heep://circos.ca) (Krzywinski et al. 2009).

\section{Results}

\section{Generation of PRV-BAC}

To perform base-editing in viral genome at plasmid DNA level, we designed and generated PRV-Ea infectious clone. The intergenic region between US9 and US2 gene was designed as F-plasmid vector (pHA2) integrating site. The transfer vector pMD18-T-US9-US2-pHA2 was constructed, linearized and co-transfected with PRV-Ea genome DNA into PK-15 cells, which was followed by plaque purification to obtain PRV-BAC recombinant virus. At the early infection phase, the self-cyclized PRV-BAC genome was isolated and transformed into $E$. coli DH10B competent cells, resulting in plasmid formed viral genome DNA (pPRV-BAC). Obviously, it is convenient to manipulate genes at plasmid level. Moreover, the F-plasmid vector was flanked by two same orientated loxP sites, thus making it simple to remove F-plasmid vector through Cre-mediated recombination (Sternberg et al. 1981) (Fig. 1A). The pPRV-BAC plasmid, wild type PRV, F-plasmid vector integrated mutant (PRV-BAC) and rescued PRV mutant (rPRV) were validated through RFLP assay with BamH I (Fig. 1B). Theoretically, the target genes in pPRV-BAC could be easily edited using CRISPR guided base-editing system in prokaryotic cells ( $\mathrm{Gu}$ et al. 2018). The CAA, CAG, CGA and TGG codons could be converted into stop codons when cytidine deamination and DNA repair occur at the target nucleotide(s) C/G (Fig. 1C).

\section{Induced Stop Mutagenesis in PRV-BAC}

To confirm the feasibility, we designed base-editing in US8 gene, which encodes 558 aa-long glycoprotein $\mathrm{E}(\mathrm{gE})$ and is involved in PRV virus cell-to-cell spread (Kratchmarov et al. 2013). After base-editing in E. coli, five colonies were cultured, harvested and taken for plasmid isolation. The US8 fragment was PCR amplified and sequenced. The Sanger sequencing results showed that all detected colonies possessed expected mutagenesis (Fig. 2A). The $46^{\text {th }}$ Gln (TGG) codon was converted into iSTOP (TAA) codon, resulting in a truncated $\mathrm{gE}$ protein. The mutant PRV-BAC plasmid was extracted and transfected into PK-15 cells, to achieve $\mathrm{gE}$ truncation virus (PRV-TgE). The morphology assay showed that truncation of $\mathrm{gE}$ significantly suppressed PRV virus intercellular transmission, thus leading to smaller plaque size (Fig. 2B, 2C). Growth kinetic studies for PRV-TgE showed that $\mathrm{gE}$ is involved in virus spread between cells, but is nonessential for virus replication (Fig. 2D). The genetic stability of PRV-TgE was analyzed by passaging and Sanger sequencing. The results showed that the iSTOP mutation was stable within 10 passages (Supplementary Figure S1). In addition, we performed induced STOP at $39^{\text {th }}$ and $83^{\text {rd }}$ codon sites in UL34 gene that encodes 261 aa-long protein (Fig. 3A). For each site, five colonies were taken for PCR and sequencing. The editing frequencies in both designed sites were $100 \%$. Besides the desired base conversion, synonymous mutations were carried out at the $38^{\text {th }}, 81^{\text {st }}$, and $82^{\text {nd }}$ codons
A

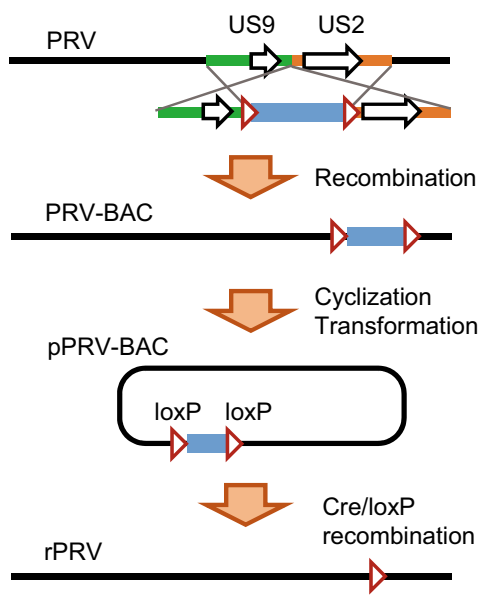

B

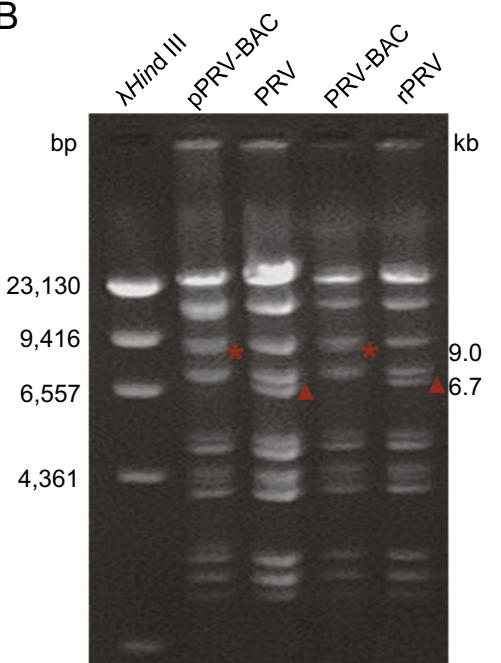

C

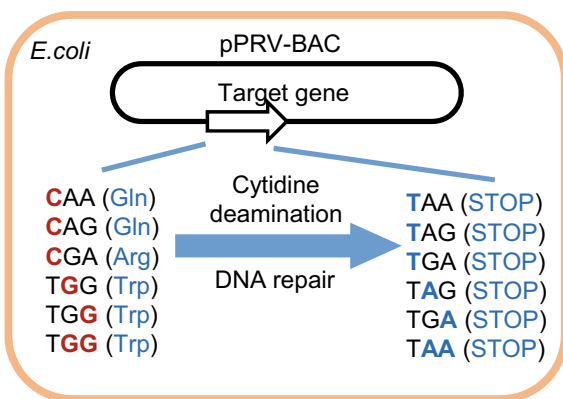

Fig. 1 Schematic representation of CRISPR/Cas9 guided base-editing in viral genome based on bacterial artificial chromosome (BAC). A Schematic representation of PRV-BAC constructing process. B RFLP analysis of pPRV-BAC, PRV, PRV-BAC, and rPRV. Viral genome DNA was digested with BamH I. The affected fragments were labeled with $*$ and $\boldsymbol{\Delta}$, which indicated $9.0 \mathrm{~kb}$ and $6.7 \mathrm{~kb}$, respectively. C Schematic representation of induced STOP in pPRVBAC mediated by BE3 in E. coli cells. 

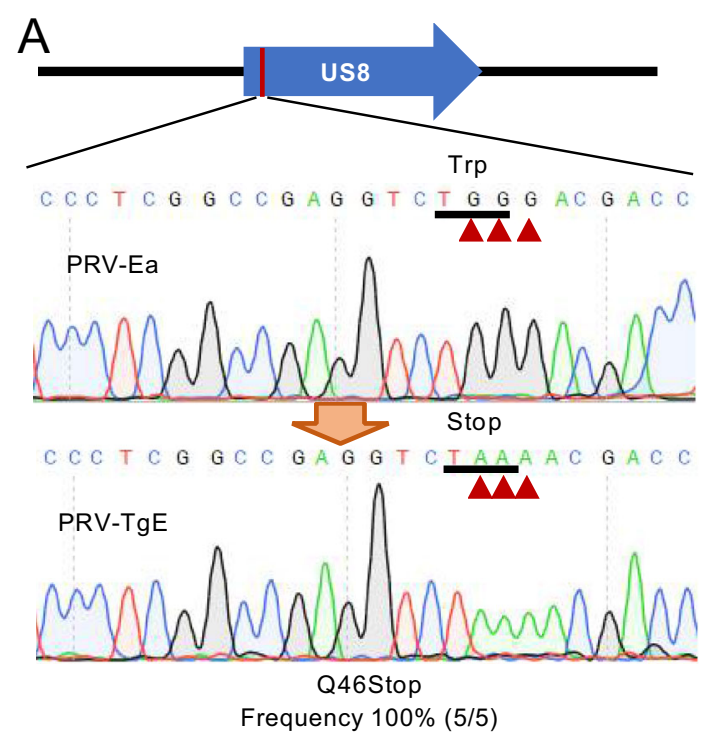

C

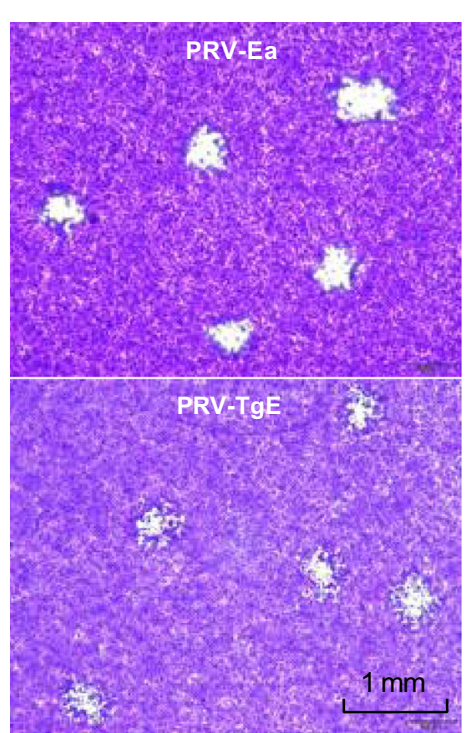

B

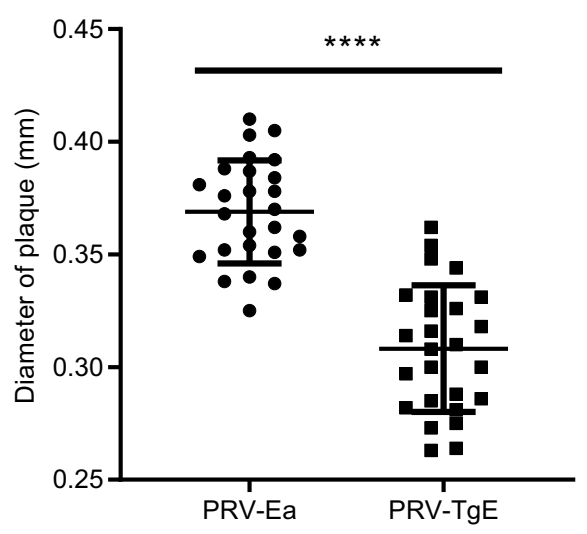

Fig. 2 BE3 mediated induction of STOP in US8 gene (coding gE protein). A Sanger sequencing of BE3 induced mutagenesis in US8 gene. The substituted bases were marked with red arrows. B The impact of $\mathrm{gE}$ truncation on plaque size. Statistical analysis was

(Fig. 3B). The typical editing window is position $4-8$ of guide RNA target sequence (counting the end distal to PAM as position 1) in eukaryotic cells (Komor et al. 2016), whereas it could be position 2-11 in some target sites in prokaryotic cells (Zheng et al. 2018). At target site 2 in UL34, the cytosine at position 2 was also edited (Fig. 3B).

\section{Prediction of Editable Sites in PRV Genome}

Next, we performed comprehensive bioinformatic analysis to gain overall insights into targetable induced stop (iSTOP) codons of PRV-Ea genome. Besides original Cas9 performed with a two-way t-test. ****, $\mathrm{P}<0.0001$. C Morphogeny assay of wild type strain PRV-Ea and gE truncation strain PRV-TgE. D Growth kinetics analysis for PRV-Ea and PRV-TgE. Detailed procedure was described in Materials and Methods.

formed BE3, a series BE systems were developed based on diverse CRIPSR-associated proteins, which recognized deviant PAM (Kim et al. 2017b; Li et al. 2018). These BE variants include VQE-BE3, EQR-BE3, VRER-BE3, SaBE3, SaKKH-BE3 and Cpf1-BE, which recognize NGAN, NGAG, NGCG, NNGRRT, NNNRRT and TTTN, respectively (Kim et al. 2017b; Li et al. 2018). Due to high GC content of herpesvirus genome, the Cpf1-BE preferring T-rich PAM was not taken for iSTOP codon analysis. A total of 68 ORFs were taken for iSTOP targeting scanning. Almost all ORFs (62/68) contain at least one iSTOP codon candidate (Supplementary Table S2). Among iSTOP codon 


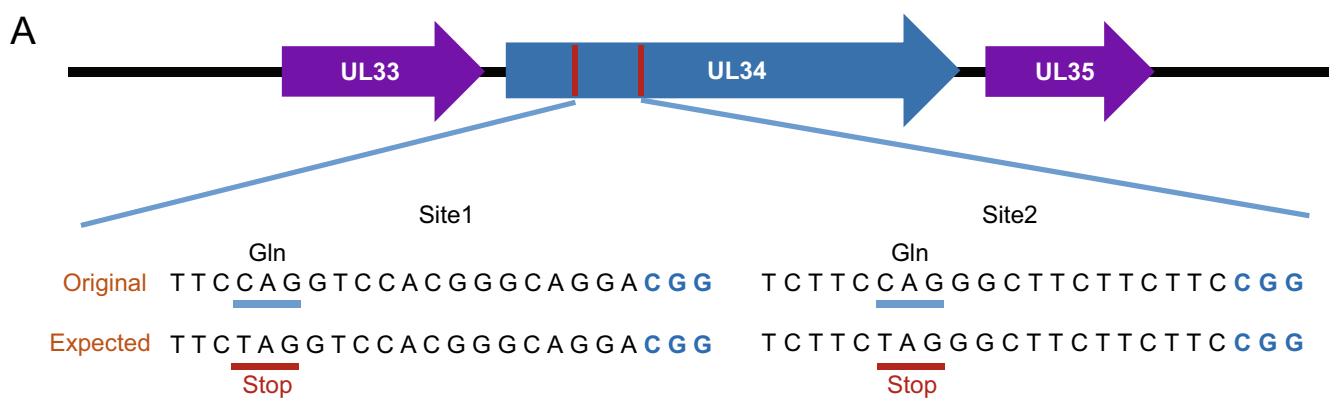

B
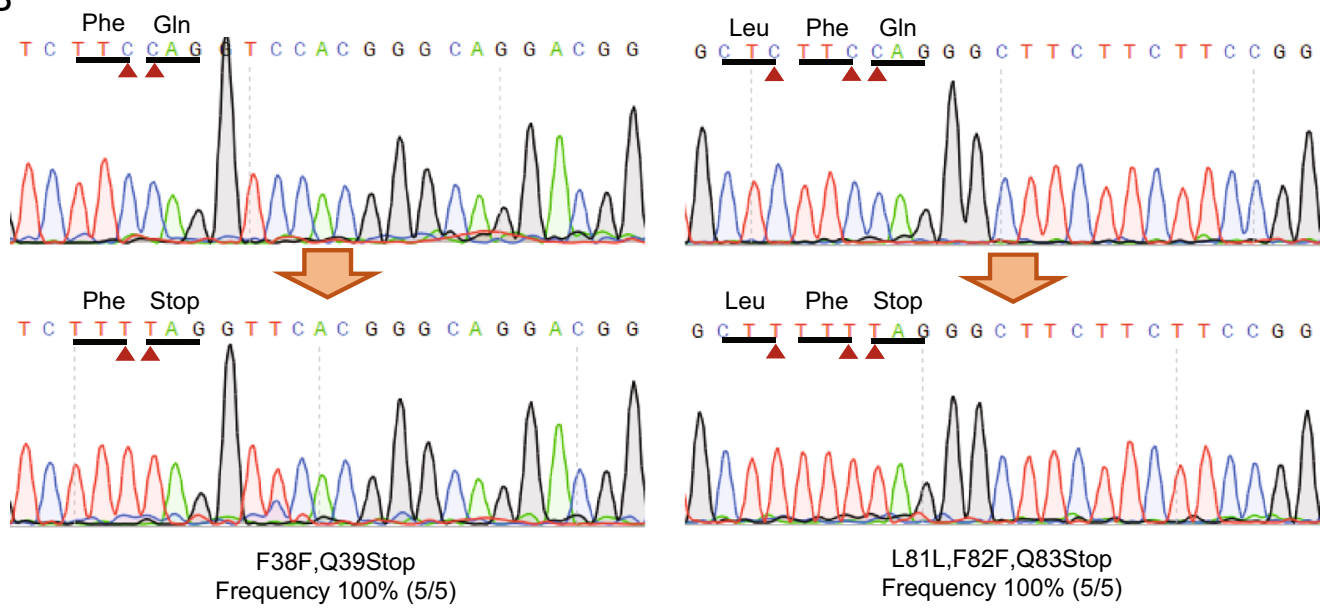

Fig. 3 BE3 mediated nucleotide conversion in UL34 gene. A Scheme of designed editing sites in UL34. Protospacer adjacent motifs (PAMs) were highlighted with blue. B Sanger sequencing of BE3 induced base-editing in UL34 gene. The substituted bases were marked with red arrows.

candidates, $66 \%$ of candidates are located within first $20 \%$ in ORFs (Fig. 4A). About 40\% (658/1643) of possibly iSTOP codons (CAAIGln, CAGIGln, CGAlArg, and TGGITrp) are targetable. The dominant potent iSTOP codons are CAGs, more than half (543/827) of which are targetable (Fig. 4B and Supplementary Table S3). Since rAPOBEC1, cytidine deaminase used in BE3 system, shows substrate bias $\mathrm{TC} \geq \mathrm{CC} \geq \mathrm{AC} \geq \mathrm{GC}$ in vitro (Komor et al. 2016), we analyzed upstream base of C. The pie chart in Fig. 4B shows that $44.1 \%$ of substrates are TC and $\mathrm{CC}$, indicating they are preferred and would be edited with high efficiency. In addition, CDA1-BE3 and AID-BE3, formed by cytidine deaminase homologs of rAPOBEC1, shows higher editing efficiencies over BE3 for AC and GC substrates (Komor et al. 2017). This information provides a guide for BE system selection for highly efficient editing.

The distribution of iSTOP codon candidates across the PRV-Ea genome is shown in Fig. 4C. We also analysed iSTOP codons in PRV-Berker strain and new-emerging PRV-HNX strain genomes. The results reveal more targetable iSTOP codons exist in both virus variants genomes (Supplementary Table S4-S5). Taken together, the bioinformatic analysis revealed that numerous coding genes in PRV genome could be truncated using BE3 system-mediated iSTOP method.

\section{Discussion}

In this study, we developed a highly efficient method to manipulate viral gene at bacterial artificial chromosome level. The plasmid formed infectious clone was conveniently and easily edited using CRISPR-guided base-editing system. The editing efficiency of BAC plasmid was $100 \%$, which is consistent with editing in bacterial genome in previous study (Gu et al. 2018; Zheng et al. 2018). Because the editing is based on highly efficient base conversion mediated by CRISPR-guided cytidine deamination at plasmid, it is virus species-independent. Additionally, infectious clone is a common platform for virologists. It could be widely applied for viral genome editing by expressing BE system in E. coli strain containing infectious clone.

In previous studies, researchers used CRISPR/Cas9 system to facilitate viral gene editing. It revealed that cleavage in viral genome caused by Cas9 protein could significantly increase recombination frequency, thus improving editing efficiency (Xu et al. 2015; Yuan et al. 2015; Liang et al. 2016). Viral genome rapidly replicates in host cells, making it difficult to manipulate viral genes using CRISPR/Cas9 system in high copy number genomes. Therefore, it is essential to select appropriate method. For example, it was too difficult to isolate recombinant viruses 
A

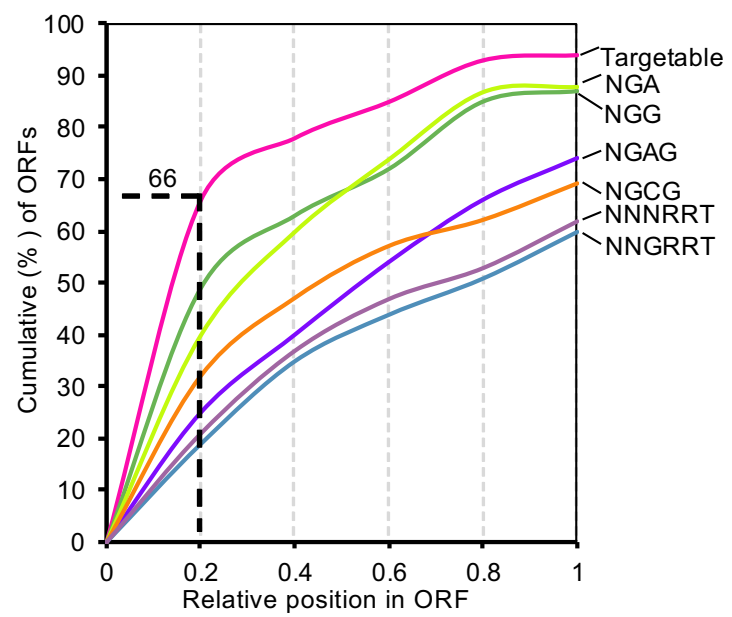

C

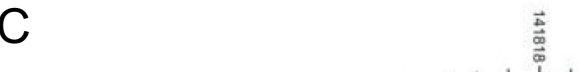

B

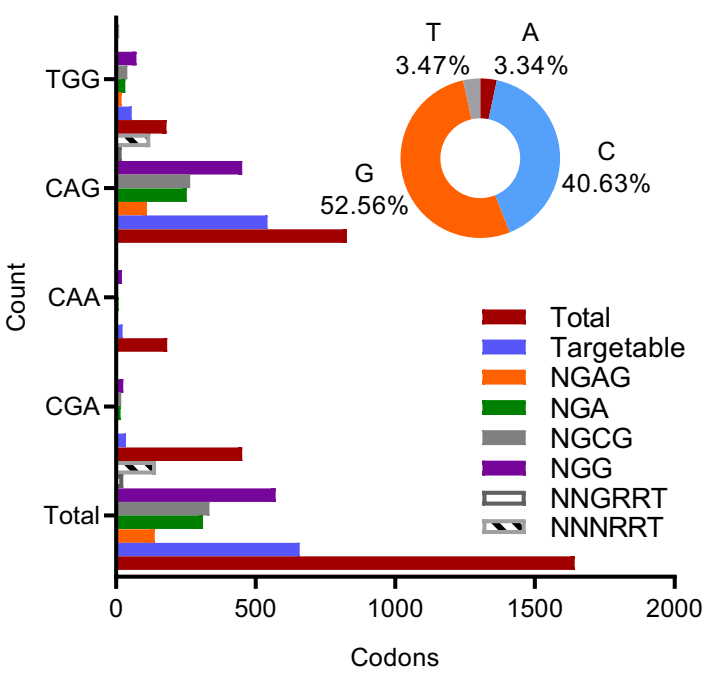

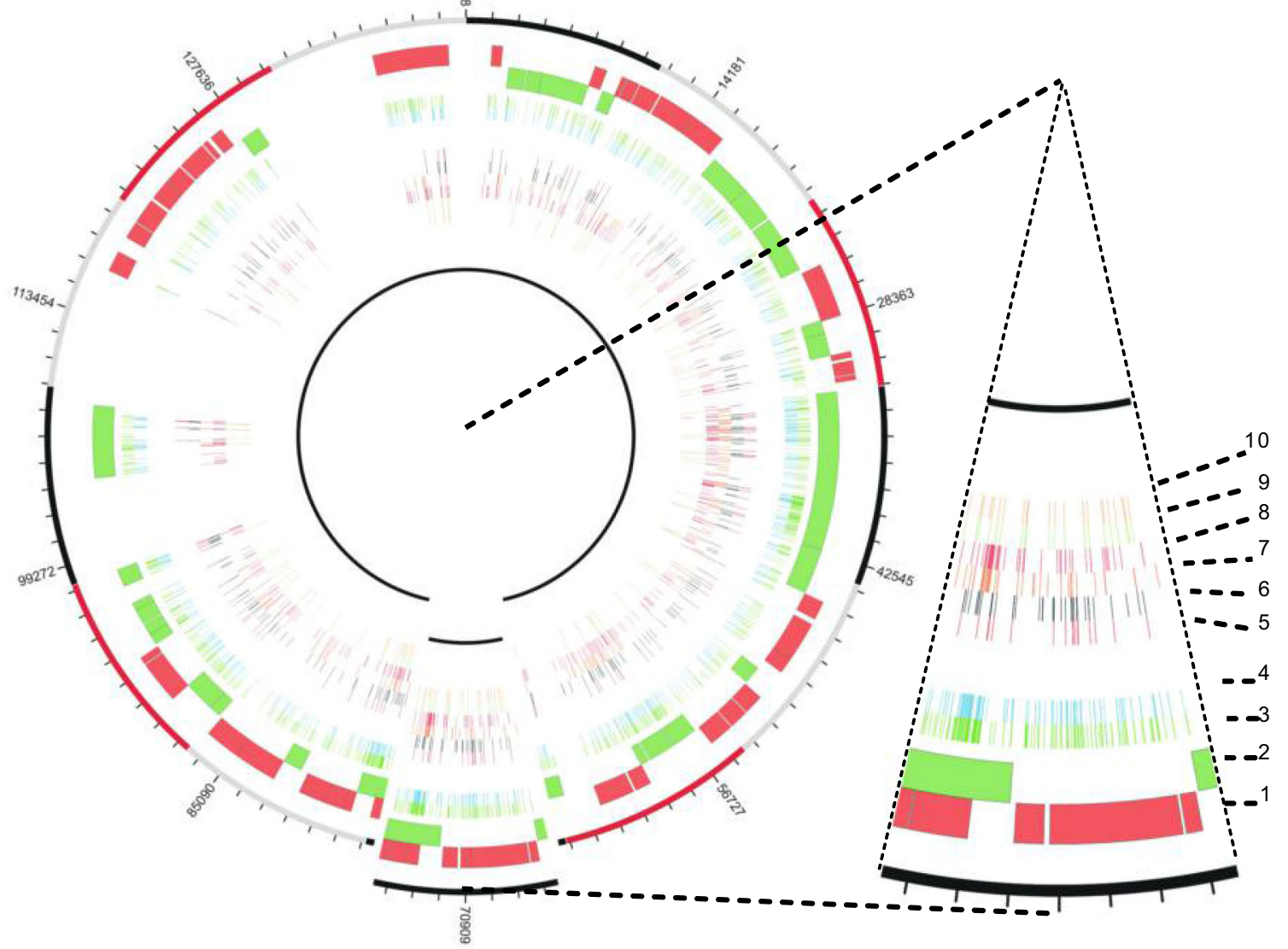

Fig. 4 Comprehensive detection of iSTOP codon candidates in PRVEa genome. A Relative position of the earliest iSTOP condon candidates in PRV-Ea ORFs (cumulative percentage) by BE3 variants with distinct PAM specificities. B Overview of TGG, CAG, CAA and CGA iSTOP codon candidates targetable by BE3 variants in PRV-Ea genome. The pie chart represented upstream nucleotide distribution of substrate cytidine. C Circos plot representing the distribution of iSTOP codon candidates targetable by BE3 in PRV-Ea genome.
Concentric circles from the outside to the inside: (1) open reading frames (ORFs) in forward strand of genome, (2) ORFs in reverse strand of genome; (3) total iSTOP codon candidates; (4) iSTOP codon candidates targetable by BE3 variants; (5-10) iSTOP codon candidates targetable by BE3 variants (EQE-BE3 (PAM: NGAG), VQRBE3 (PAM: NGAN), VRER-BE3 (PAM: NGCG), BE3 (PAM: NGG), SaBE3 (PAM: NNGRRT), and SaKKH-BE3 (PAM: NNNRRT), respectively). 
using transfection followed by infection method. Cotransfection of CRISPR/Cas9 expression plasmid and PRV genome resulted in higher recombination rate and made it possible to obtained recombination virus (Xu et al. 2015). On the other hand, it is essential to avoid wild type virus contamination during isolation process due to viruses spread and infect cells easily. The flowcytometry based on fluorescence reporter gene simplified and facilitated isolation of recombination virus (Liang et al. 2016). Bacterial artificial chromosome method has been employed to simplify genome editing procedure, which significantly improved the recombination rate (Adler et al. 2000; Almazán et al. 2008; Liu et al. 2008; Zou et al. 2014; Feng et al. 2015; Zou et al. 2015; Richards et al. 2016; Close et al. 2017). Besides lambda Red mediated recombination (Liu et al. 2008), the CRISPR-guided base-editing method provided an alternative approach for viral gene editing on BAC platform. Our strategy could directly edit nucleotide at target loci without donor DNA and achieve absolutely high editing rate with appropriate manner. In addition, base-editing performs at BAC DNA level, indicating that the CRISPR-guided base-editing system is virus speciesindependent and plaque purification of mutant virus is not required. Our results showed that PRV-TgE mutant was stable at least 10 passages, indicating that this method might be utilized for the development of attenuated live vaccines. Previous studies demonstrated that PRV deletion mutants lacking pUL34 produced infectious progeny at 3to 4-log-reduced titer compared to wild-type PRV (Klupp et al. 2000, 2007; Fuchs et al. 2002). Currently, we do not have UL34-expressing cell line to rescue PRV UL34 mutant. We transfected PK-15 cells with plasmid pPRV BAC harboring $U L 34$ point mutations for three times, but failed to achieve PRV UL34 mutant. Thus, we did not analyse the plaque size and growth kinetics. Of notice, not all viral genomes have been or can be cloned as infectious plasmids. Whether the base-editing systems can be readapted for direct targeting of DNA viruses during their replication in eukaryotic cells remains to be investigated.

Acknowledgements This work was supported by the National Key Research and Development Program (2016YFD0500105) and the Natural Science Foundation of China (31770191). We thank Professor Mei-Lin Jin for providing Cre expression plasmid.

Author Contributions ZFL and KZ designed the experiments; KZ performed genome editing in PRV BAC; XW and YXC constructed the PRV BAC; FFJ and LS investigated characteristic of PRV mutant; HCC provided PRV; KZ, and ZFL wrote and revised the manuscript.

\section{Compliance with Ethical Standards}

Conflict of interest The authors declare that they have no conflict of interest.
Animal and Human Rights Statement This article does not contain any studies with human or animal subjects performed by any of the authors.

\section{References}

Adler H, Messerle M, Wagner M, Koszinowski UH (2000) Cloning and mutagenesis of the murine gammaherpesvirus 68 genome as an infectious bacterial artificial chromosome. J Virol 74:6964-6974

Almazán F, Galán C, Enjuanes L (2008) Engineering infectious cDNAs of coronavirus as bacterial artificial chromosomes. Methods Mol Biol 454:275-291

Billon P, Bryant EE, Joseph SA, Nambiar TS, Hayward SB, Rothstein R, Ciccia A (2017) CRISPR-mediated base editing enables efficient disruption of eukaryotic genes through induction of STOP codons. Mol Cell 67(1068-1079):e1064

Bull JJ (2015) Evolutionary reversion of live viral vaccines: Can genetic engineering subdue it? Virus Evol 1:pii: vev005

Chen H, Fang L, He Q, Jin M, Suo X, Wu M (1998) Study on the isolation and identification of the EA strain of pseudorabies virus. Chin J Anim Vet 29:156-161 (in Chinese)

Close WL, Bhandari A, Hojeij M, Pellett PE (2017) Generation of a novel human cytomegalovirus bacterial artificial chromosome tailored for transduction of exogenous sequences. Virus Res 242:66-78

Court DL, Sawitzke JA, Thomason LC (2002) Genetic engineering using homologous recombination. Annu Rev Genet 36:361-388

Feng C, Bluhm BH, Correll JC (2015) Construction of a Spinach Bacterial Artificial Chromosome (BAC) Library as a resource for gene identification and marker development. Plant Mol Biol Rep 33:1996-2005

Fuchs W, Klupp BG, Granzow H, Osterrieder N, Mettenleiter TC (2002) The interacting UL31 and UL34 gene products of pseudorabies virus are involved in egress from the host-cell nucleus and represent components of primary enveloped but not mature virions. J Virol 76:364-378

Gasiunas G, Barrangou R, Horvath P, Siksnys V (2012) Cas9-crRNA ribonucleoprotein complex mediates specific DNA cleavage for adaptive immunity in bacteria. Proc Natl Acad Sci USA 109:E2579-E2586

Gaudelli NM, Komor AC, Rees HA, Packer MS, Badran AH, Bryson DI, Liu DR (2017) Programmable base editing of $A * T$ to $G * C$ in genomic DNA without DNA cleavage. Nature 551:464-471

Ge S, Li J, Fan X, Liu F, Li L, Wang Q, Ren W, Bao J, Liu C, Wang H, Liu Y, Zhang Y, Xu T, Wu X, Wang Z (2018) Molecular characterization of African Swine Fever Virus, China, 2018. Emerg Infect Dis 24:2131-2133

Gu TN, Zhao SQ, Pi YS, Chen WZ, Chen CY, Liu Q, Li M, Han DL, Ji QJ (2018) Highly efficient base editing in Staphylococcus aureus using an engineered CRISPR RNA-guided cytidine deaminase. Chem Sci 9:3248-3253

Jinek M, Chylinski K, Fonfara I, Hauer M, Doudna JA, Charpentier E (2012) A programmable dual-RNA-guided DNA endonuclease in adaptive bacterial immunity. Science 337:816-821

Kim K, Ryu S-M, Kim S-T, Baek G, Kim D, Lim K, Chung E, Kim S, Kim J-S (2017a) Highly efficient RNA-guided base editing in mouse embryos. Nat Biotechnol 35:435-437

Kim YB, Komor AC, Levy JM, Packer MS, Zhao KT, Liu DR (2017b) Increasing the genome-targeting scope and precision of base editing with engineered Cas9-cytidine deaminase fusions. Nat Biotechnol 35:371-376 
Klupp BG, Granzow H, Mettenleiter TC (2000) Primary envelopment of pseudorabies virus at the nuclear membrane requires the UL34 gene product. J Virol 74:10063-10073

Klupp BG, Granzow H, Fuchs W, Keil GM, Finke S, Mettenleiter TC (2007) Vesicle formation from the nuclear membrane is induced by coexpression of two conserved herpesvirus proteins. Proc Natl Acad Sci USA 104:7241-7246

Komor AC, Kim YB, Packer MS, Zuris JA, Liu DR (2016) Programmable editing of a target base in genomic DNA without double-stranded DNA cleavage. Nature 533:420-424

Komor AC, Zhao KT, Packer MS, Gaudelli NM, Waterbury AL, Koblan LW, Kim YB, Badran AH, Liu DR (2017) Improved base excision repair inhibition and bacteriophage $\mathrm{Mu}$ Gam protein yields C:G-to-T: A base editors with higher efficiency and product purity. Sci Adv 3:eaao4774

Koonin EV, Senkevich TG, Dolja VV (2006) The ancient virus world and evolution of cells. Biol Direct 1:29

Kratchmarov R, Kramer T, Greco TM, Taylor MP, Ch'ng TH, Cristea IM, Enquist LW (2013) Glycoproteins gE and gI are required for efficient KIF1A-dependent anterograde axonal transport of alphaherpesvirus particles in neurons. J Virol 87:9431-9440

Krzywinski M, Schein J, Birol I, Connors J, Gascoyne R, Horsman D, Jones SJ, Marra MA (2009) Circos: an information aesthetic for comparative genomics. Genome Res 19:1639-1645

Li X, Wang Y, Liu Y, Yang B, Wang X, Wei J, Lu Z, Zhang Y, Wu J, Huang X, Yang L, Chen J (2018) Base editing with a Cpf1cytidine deaminase fusion. Nat Biotechnol 36:324-327

Liang X, Sun L, Yu T, Pan Y, Wang D, Hu X, Fu Z, He Q, Cao G (2016) A CRISPR/Cas9 and Cre/Lox system-based express vaccine development strategy against re-emerging Pseudorabies virus. Sci Rep 6:19176

Liu Z, Chen H, He Q, Zhou F, Fang L (2002) Construction of pseudorabies virus Ea TK-/gE-/gp63-mutant strain and the study on its biological property. Wei Sheng Wu Xue Bao 42:370-374 (in Chinese)

Liu ZF, Brum MC, Doster A, Jones C, Chowdhury SI (2008) A bovine herpesvirus type 1 mutant virus specifying a carboxylterminal truncation of glycoprotein $\mathrm{E}$ is defective in anterograde neuronal transport in rabbits and calves. J Virol 82:7432-7442

Ma Y, Zhang J, Yin W, Zhang Z, Song Y, Chang X (2016) Targeted AID-mediated mutagenesis (TAM) enables efficient genomic diversification in mammalian cells. Nat Methods 13:1029-1035

Meyer AG, Wilke CO (2015) Geometric constraints dominate the antigenic evolution of influenza H3N2 hemagglutinin. PLoS Pathog 11:e1004940

Nishida K, Arazoe T, Yachie N, Banno S, Kakimoto M, Tabata M, Mochizuki M, Miyabe A, Araki M, Hara KY (2016) Targeted nucleotide editing using hybrid prokaryotic and vertebrate adaptive immune systems. Science 353:aaf8729

Peiris JS, Chu CM, Cheng VC, Chan KS, Hung IF, Poon LL, Law KI, Tang BS, Hon TY, Chan CS, Chan KH, Ng JS, Zheng BJ, Ng WL, Lai RW, Guan Y, Yuen KY, Group HUSS (2003) Clinical progression and viral load in a community outbreak of coronavirus-associated SARS pneumonia: a prospective study. Lancet 361:1767-1772

Peng JM, Wang Y, Tian ZJ, Zou YJ, Chen J, An TQ, Tong GZ (2009) Construction of a recombinant pseudorabies virus with BAC plasmid insertion in the TK gene. Chin Prev Vet Med 31:10-15 (in Chinese)

Pomeranz LE, Reynolds AE, Hengartner CJ (2005) Molecular biology of pseudorabies virus: impact on neurovirology and veterinary medicine. Microbiol Mol Biol Rev 69:462-500
Post LE, Thomsen DR, Petrovskis EA, Meyer AL, Berlinski PJ, Wardley RC (1990) Genetic engineering of the pseudorabies virus genome to construct live vaccines. J Reprod Fertil Suppl 41:97-104

Richards AL, Sollars PJ, Smith GA (2016) New tools to convert bacterial artificial chromosomes to a self-excising design and their application to a herpes simplex virus type 1 infectious clone. BMC Biotechnol 16:64

Smith GA, Enquist LW (2000) A self-recombining bacterial artificial chromosome and its application for analysis of herpesvirus pathogenesis. Proc Natl Acad Sci USA 97:4873-4878

Sternberg N, Hamilton D, Hoess R (1981) Bacteriophage P1 sitespecific recombination. II. Recombination between loxP and the bacterial chromosome. J Mol Biol 150:487-507

Wang J, Osterrieder N (2011) Generation of an infectious clone of duck enteritis virus (DEV) and of a vectored DEV expressing hemagglutinin of $\mathrm{H} 5 \mathrm{~N} 1$ avian influenza virus. Virus Res 159:23-31

Wang H, La Russa M, Qi LS (2016) CRISPR/Cas9 in genome editing and beyond. Annu Rev Biochem 85:227-264

Wang J, Guo R, Qiao Y, Xu M, Wang Z, Liu Y, Gu Y, Liu C, Hou J (2016) An inactivated $g E$-deleted pseudorabies vaccine provides complete clinical protection and reduces virus shedding against challenge by a Chinese pseudorabies variant. BMC Vet Res $12: 277$

Wang X, Wu CX, Song XR, Chen HC, Liu ZF (2017) Comparison of pseudorabies virus China reference strain with emerging variants reveals independent virus evolution within specific geographic regions. Virology 506:92-98

Wang T, Tong W, Ye C, Yu Z, Chen J, Gao F, Shan T, Yu H, Li L, Li G, Tong G, Zheng H (2018) Construction of an infectious bacterial artificial chromosome clone of a pseudorabies virus variant: reconstituted virus exhibited wild-type properties in vitro and in vivo. J Virol Methods 259:106-115

Wu R, Bai C, Sun J, Chang S, Zhang X (2013) Emergence of virulent pseudorabies virus infection in northern China. J Vet Sci $14: 363-365$

Xu A, Qin C, Lang Y, Wang M, Lin M, Li C, Zhang R, Tang J (2015) A simple and rapid approach to manipulate pseudorabies virus genome by CRISPR/Cas9 system. Biotechnol Lett 37:1265-1272

Yuan M, Zhang WS, Wang J, Al Yaghchi C, Ahmed J, Chard L, Lemoine NR, Wang YH (2015) Efficiently editing the vaccinia virus genome by using the CRISPR-Cas9 system. J Virol 89:5176-5179

Zheng K, Wang Y, Li N, Jiang FF, Wu CX, Liu F, Chen HC, Liu ZF (2018) Highly efficient base editing in bacteria using a Cas9cytidine deaminase fusion. Commun Biol 1:32

Zhou X, Li N, Luo Y, Liu Y, Miao F, Chen T, Zhang S, Cao P, Li X, Tian K, Qiu H, Hu R (2018) Emergence of African Swine Fever in China, 2018. Transbound Emerg Dis 65:1482-1484

Zong Y, Wang Y, Li C, Zhang R, Chen K, Ran Y, Qiu J-L, Wang D, Gao C (2017) Precise base editing in rice, wheat and maize with a Cas9-cytidine deaminase fusion. Nat Biotechnol 35:438-440

Zou Z, Liu Z, Jin M (2014) Efficient strategy to generate a vectored duck enteritis virus delivering envelope of duck Tembusu virus. Viruses 6:2428-2443

Zou Z, Hu Y, Liu Z, Zhong W, Cao H, Chen H, Jin M (2015) Efficient strategy for constructing duck enteritis virus-based live attenuated vaccine against homologous and heterologous H5N1 avian influenza virus and duck enteritis virus infection. Vet Res $46: 1-15$ 